\title{
Corneal Spheres derived from Human Embryonic and Human Pluripotent Parthenogenetic Stem Cells
}

Alina Ostrowska1*, Jackie Cochran ${ }^{2}$, Larissa Agapova ${ }^{1}$, Amber Buz'Zard $^{1}$, Nikolay Turovets ${ }^{1 \#, ~ J e r e m y ~ H a m m o n d ~}{ }^{2}$, Irina Turovets ${ }^{1}$, Subramanian Krishnakumar ${ }^{3}$, Andrey Semechkin ${ }^{1}$, Judith Kelleher-Andersson ${ }^{5}$, Jeffrey Janus ${ }^{1}$ and Marie Csete ${ }^{4}$

${ }^{1}$ International Stem Cell Corporation, Carlsbad CA 92008

${ }^{2}$ Lifeline Cell Technology, Frederick MD

${ }^{3}$ Vision Research Foundation, Sankara Nethralaya, India

${ }^{4}$ University of California San Diego, Dept. of Anesthesiology, San Diego CA 92103

${ }^{5}$ Neuro Nasent Inc., Clarksville, MD, 21029

\#NT Consulting, Carlsbad CA 92011

\begin{abstract}
Corneal blindness is common. Cornea transplants are the most commonly performed organ transplants, but the need for corneal grafts worldwide far outweighs the supply of healthy donor corneas. Here we describe a differentiation protocol that yields corneal orbs from human embryonic stem cells (hESC) as well as from human pluripotent parthenogenetic stem cells (hpSC), and therefore can be manufactured free of transmissible pathogens. Cornea and other tissues generated from parthenogenetic stem cells that are homozygous at HLA loci have a distinct immunologic advantage over fully allogeneic grafts, and this report is the first to describe multilayered cornea generated from hpSC. The differentiated corneal product is layered and anatomically similar to normal human cornea, expresses appropriate corneal markers at the mRNA and protein (and secreted protein) levels, and is permeable to topical ophthalmic drugs. This 3D stem cell-derived cornea is a foundational step in development of appropriately organized, functional corneal grafts from $\mathrm{hESC}$ and $\mathrm{hpSC}$ for use in in vitro assays as well as regenerative therapies.
\end{abstract}

\section{Introduction}

The cornea is more than a protective shield, it also serves as an external lens and is therefore critical for refraction and normal vision. An estimated 8 to 10 million worldwide are blind from corneal disease [1]. In developed countries, corneas are the most common 'organ' transplants, but a significant unmet medical need for corneal grafts persists. Fully artificial corneas have helped significant numbers of patients with severe corneal disease or injury, but do not meet the medical needs of most patients requiring corneal grafting, and so have limited impact on the need for donor corneas [2]. Stem cell-derived corneas, free of transmissible pathogens, represent a promising and safe alternative to cadaveric grafts, provided they can be generated costeffectively, with function (including barrier, absorption, refraction) equivalent to that afforded by cadaveric grafts. Here we describe a novel multilayer corneal structure generated from human pluripotent stem cells, a first step in the goal of developing a full-thickness corneal graft from a single differentiation protocol.

The cornea is the clear (transparent) front part of the eye and is comprised of three main cellular layers - an outer non-keratinized stratified epithelium, middle stromal layer containing a hydrated extracellular matrix (ECM) with scattered corneal fibroblasts (keratocytes), and an inner, single-cell endothelial layer. Each layer is separated by distinct, specialized basal lamina. Bowman's layer is most anterior, located between the epithelium and the stroma. Descemet's membrane separates the stroma and the endothelium, serving as a basement membrane for corneal endothelium [3]. Advances in cornea engineering techniques have just started as another important alternative to overcome the limitation of 3D-corneal tissue available for transplantation $[7,29]$. To this end, bioengineered corneas to replace partial or full-thickness corneal defects have recently been developed as an alternative to cadaveric grafts [6,7] and an acellular collagen-based cornea was recently tested in a Phase I trial [8]. Decellularization of animal corneas is another promising method for the development of artificial human corneas by tissue engineering [37].
Adult human cornea harbors resident stem cells in the limbus (between the cornea and conjunctiva) [reviewed in 4], and these limbal stem cells mediate regeneration and wound repair of the corneal epithelium throughout adult life. Recent success in transplanting (autologous, adult) limbal stem cells for corneal burns [5] points to the importance of stem cell biology for therapy of corneal disease. Limbal stem cell transplantation, both autologous and allogeneic, is a major stem cell success story and is now available in many centers, but is not useful for diseases where more than corneal epithelium is compromised; complete 3D-corneas containing all cellular compartments of the cornea are still a necessity for treatment of the many patients with corneal blindness.

Derivation of human pluripotent embryonic stem cell (hESC) lines and recent advances in hESC biology have generated great interest in the field of stem cell-based cornea engineering [9-11]. Cell culture technologies that expand undifferentiated hESCs and subsequent methods to direct differentiation into corneal-like cells are improving and open up the possibility of producing corneal constructs for use in cellular therapy. Corneal epithelial cells have been produced by culturing hESC on collagen IV using medium conditioned by limbal fibroblasts[12].

*Corresponding author: Alina Ostrowska PhD, International Stem Cell Corporation, 5950 Priestly Drive, Carlsbad CA 92008, Tel: 760-940-6383; Fax: 760-476-0600; E-mail: aostrowska@intlstemcell.com

Received November 02, 2011; Accepted December 03, 2011; Published December 05, 2011

Citation: Ostrowska A, Cochran J, Agapova L, Buz'Zard A, Turovets N, et al (2011) Corneal Spheres derived from Human Embryonic and Human Pluripoten Parthenogenetic Stem Cells. J Stem Cell Res Ther S2:006. doi:10.4172/21577633.S2-006

Copyright: (c) 2011 Ostrowska A, et al. This is an open-access article distributed under the terms of the Creative Commons Attribution License, which permits unrestricted use, distribution, and reproduction in any medium, provided the original author and source are credited. 
Our focus here is on differentiation of a whole cornea from pluripotent stem cells generally, but particularly human parthenogenetic stem cells or hpSC. hpSC are morphologically similar to hESC, express the same pluripotency markers, have high levels of alkaline phosphatase and telomerase activity, and give rise in vitro and in vivo to derivatives of all three embryonic germ layers. Unlike hESC, hpSC cannot differentiate into extraembryonic tissues $[38,39]$. Different activation techniques during isolation of hpSC from unfertilized eggs allow creation of either heterozygous hpSC that are genetically identical to the oocyte donor, or HLA homozygous hpSC which are immunologically less complex [13-15]. As such, derivatives of these homozygous lines transplanted in appropriate donors will be less immunogenic than traditional cadaveric allogeneic grafts. This report represents a first but significant step toward generation of sterile, functional, less immunogenic 3D corneal grafts for use in regenerative therapies.

\section{Materials and Methods}

\section{In vitro differentiation of corneas from human pluripotent stem cells}

The WA09 hESC line was provided by Dr. Mike West. The hpSC lines hpSC-1, hpSC-3, hpSC-5 [13] and hpSC-Hhom-1 [14] were derived by Revazova et al., and are owned by International Stem Cell Corporation. Undifferentiated stem cells were maintained on a feeder layer of human Mitomycin-C treated dermal fibroblasts (MCFibs ${ }^{\mathrm{m}}$; Lifeline Cell Technology", Fredrerick, MD) in hESC Growth Medium (Knockout $^{\mathrm{m}}$ D-MEM; Invitrogen, Carlsbad, CA) supplemented with $2.4 \mathrm{mM}$ GlutaMax (Invitrogen), $0.12 \mathrm{mM}$ MEM Non-Essential Amino Acid Solution (Invitrogen), 0.12\% mercaptoethanol (Invitrogen), 8\% Plasmanate, 8\% Knockout Serum Replacement (Invitrogen) $10 \mathrm{ng} / \mathrm{ml}$ basic fibroblast growth factor (bFGF, Lifeline Cell Technology), and 10 $\mathrm{ng} / \mathrm{ml}$ leukemia inhibitory factor (LIF, Chemicon, Billerica MA).

To initiate differentiation the stem cells were allowed to grow for approximately 10 days (with medium replacement every other day) in a humidified $5 \% \mathrm{CO}_{2}$ incubator. When colonies formed (morphologically similar to burst forming units with sharp edges), both LIF and bFGF were removed from the medium, and on day 17, these differentiated cultures were passaged with Collagenase Type IV (890 U/ml; Lifeline Cell Technology) and transferred to deep 6-well plates (Greiner BioOne, Frickenhausen, Germany) for further differentiation in lowattachment conditions. The cultures were maintained for an additional 100-120 days in a humidified atmosphere of 5\% $\mathrm{CO}_{2} / 95 \%$ air, with medium added twice a week, during which the dome-like colonies grew in size and became fluid-filled clear spheres. The spheres break off from the adherent cells and float freely; these were retrieved from the medium for characterization.

\section{Histology and immunocytochemistry}

Ten corneal orb constructs derived from hESC or hpSC were used for this study. For histological analysis the corneal orbs were cut in half, each half measuring approximately $5 \times 10 \mathrm{~mm}$. One half was fixed for 12 hours in 10\% buffered formalin, embedded in paraffin and sectioned to a thickness of $5 \mu \mathrm{m}$ (small size hpSC-derived orbs were fixed in their entirety). Sections were stained with hematoxylin and eosin (H\&E), Masson's trichrome, or periodic acid Schiff (PAS) using standard staining protocols. Sections were also stained for cytokeratins, using primary AE1/3 antibody (Chemicon) for pan-cytokeratins.

For immunofluorescence assays, orb halves (or whole small size constructs) were snap-frozen in OCT compound and sectioned on a Tissue Tek II Cryostat. Frozen sections $(5 \mu \mathrm{m})$ were air-dried and fixed with cold $\left(-20^{\circ} \mathrm{C}\right)$ acetone for 10 minutes, then blocked with $1 \%$ bovine serum albumin (BSA) in PBS at room temperature for 1 hour. Sections were incubated with primary antibodies at $4^{\circ} \mathrm{C}$ overnight and subsequently with appropriate secondary antibodies at room temperature for 45 minutes. Primary antibodies were: mouse antikeratin 3, 1:50 dilution (AE5 clone, MP Biomedicals, Solon OH), mouse anti-cytokeratin 18, 1:50 (Santa Cruz Biotechnology Inc., Santa Cruz CA), mouse anti-cytokeratin 19, 1:200 (Abcam, Cambridge MA), rabbit anti-connexin-43, 1:100 (Invitrogen), rabbit anti-mucin-1, 1:100 (Abcam), rabbit anti-zona occludens (ZO-1), 1:50 (Invitrogen). Secondary donkey anti-mouse or anti-rabbit antibodies conjugated with AlexaFluor 546 (Invitrogen) were used. For vimentin staining, fixed tissue sections were embedded in paraffin and microwaved for antigen retrieval. Slides were washed briefly in PBS and then after blocking and permeabilization, treated with primary monoclonal antibody (Clone LN-6, Sigma-Aldrich, St. Louis MO). After labeling with the appropriate secondary antibodies, and nuclear counterstain with 4',6-diamidino-2-phenylindole (DAPI), or propidium iodide, all sections were examined using fluorescence microscopy. Positive controls for these studies were cadaveric human corneas.

\section{Preparation of human cornea samples}

Non-diseased banked human corneas $(n=2)$ were obtained from San Diego Eye Bank. The eyes were procured and processed using criteria established by the Medical Standards of the Eye Bank Association of America and the U.S. Food \& Drug Administration.

\section{Real-time quantitative PCR (RT-qPCR)}

Stem cell-derived corneal constructs were examined for expression of normal human cornea genes using real-time quantitative PCR (RTqPCR). Total RNA was extracted using the QIAsymphony automatic purification system, according to the manufacturer's instructions (Qiagen, Valencia CA), and 100-500 ng total RNA was used for reverse transcription with the iScript ${ }^{\text {tix }} c D N A$ synthesis kit (Bio-Rad,). PCR reactions were run in duplicate using $1 / 40$-th of the cDNA per reaction and $400 \mathrm{nM}$ forward and reverse primers or the QuantiTect Primer Assay, together with Quantitest SYBR' Green master mix (Qiagen). Real-time PCR reactions were run on the Rotor-Gene Q (Qiagen). Relative quantification was performed against a standard curve and normalized to the expression levels of one of the following housekeeping genes: cyclin G (CYCG), beta-glucuronidase (GUSB) or TATA box binding protein (TBP). After normalization, the samples were plotted relative to the first sample in the data set and the standard deviation of the expression measurements was calculated. The source of primers is shown are shown in Table 1. RNA isolated from human donor cornea for use as a comparison for gene expression of stem cellderived corneas.

\section{Drug permeability of stem cell-derived corneas}

Corneal disease is often treated with topical drugs, possible because the normal cornea is permeable and allows rapid absorption [reviewed in 16]. The goal of these first permeability studies was qualitative, to test whether compounds with known high and low absorption behaved as expected relative to each other when applied to the differentiated corneal orbs. To test permeability function, intact corneal orbs were incubated with test compounds $(10 \mu \mathrm{M})$ at $37^{\circ} \mathrm{C}$ for $30 \mathrm{~min}$. Two model compounds were used - atenolol and antipyrine, as low- and highpermeability reference compounds, respectively. After incubation, the 
fluid inside the orbs was carefully collected by needle aspiration, and used to measure drug concentrations by LC-MS/MS. The apparent permeability coefficient $\left(\mathrm{P}_{\text {app }}\right)$, a parameter commonly used to express in vitro permeability across a cell monolayer or tissue, was calculated as: $\mathrm{P}_{\text {app }}=\mathrm{Q} /\left(\mathrm{C}_{0} \times \mathrm{A} \times \mathrm{T}\right)$, where $\mathrm{Q}$ is the amount of drug accumulated in the orbs, $\mathrm{C}_{0}$ is the applied drug concentration, $\mathrm{A}$ is the surface area of the orbs, and $\mathrm{T}$ is the incubation time.

\section{Determination of growth factor levels in differentiating cornea conditioned medium}

Commercial TGF- $\beta 1$ and EGF ELISA immunoassay kits (Invitrogen) were used for quantitation of these factors in conditioned medium from cultured corneal constructs at different stages of differentiation. The conditioned medium was diluted 1:1 with serumfree DMEM, and assayed according to kit manufacturer's protocols.

\section{Results}

\section{Differentiation and morphology of stem cell-derived corneal orbs}

The typical early appearance of cells that differentiated into corneal orbs was as small, tightly packed colonies in which individual cells were round, in comparison to the spindle-shaped feeder layer cells (Figure 1A). Often a few healthy stem cell colonies were noticeable the first day after low density inoculation, and became more heterogenous in shape, containing flattened cells with epithelial morphology, attached to the culture dishes. (Figure 1B,C). After 5-7 days more of differentiation, the colonies became multilayered, dense clusters in which individual cells were indistinguishable by light microscopy. In some colonies, a distinct clear spherical (orb-shaped) dome was observed, indicating the formation of a more mature cornea-like structure (Figure 1D). On day 17-19 the colonies were subcultured onto deep well plates under low attachment conditions, and within the next 3-4 weeks, freely floating pellucid spheres (corneal orbs) could be observed under a dissecting microscope. The orbs could be seen by eye at approximately 50-day culture, with diameters of 1-2 mm (Figure 1E). Spheres with diameters as large as 9-15 mm developed over 120 days (Figure 1F,G), however the orbs generated from $\mathrm{hpSC}$ were significantly smaller and reached diameters

\begin{tabular}{|l|l|l|}
\hline Gene & Cat\# & Producer \\
\hline CK8 & QT01847741 QuantiTect Primer Assay & Qiagen \\
\hline CK10 & QT00017045 QuantiTect Primer Assay & Qiagen \\
\hline CK18 & QT01846327 QuantiTect Primer Assay & Qiagen \\
\hline Collagen5 & QT00044527 QuantiTect Primer Assay & Qiagen \\
\hline Connexin43 & QT00012684 QuantiTect Primer Assay & Qiagen \\
\hline Decorin & QT00032459 QuantiTect Primer Assay & Qiagen \\
\hline Biglycan & QT01870029 QuantiTect Primer Assay & Qiagen \\
\hline Importin 13 & QT00031157 QuantiTect Primer Assay & Qiagen \\
\hline Integrin alpha 9 & QT01667743 QuantiTect Primer Assay & Qiagen \\
\hline Keratocan & QT00021280 QuantiTect Primer Assay & Qiagen \\
\hline Lumican & QT00058982 QuantiTect Primer Assay & Qiagen \\
\hline Mucin 1 & QT00015379 QuantiTect Primer Assay & Qiagen \\
\hline Enolase alpha & QT01681722 QuantiTect Primer Assay & Qiagen \\
\hline ZO-1 & QT00077308 QuantiTect Primer Assay & Qiagen \\
\hline Table 1: & & \\
\hline
\end{tabular}

Table 1:
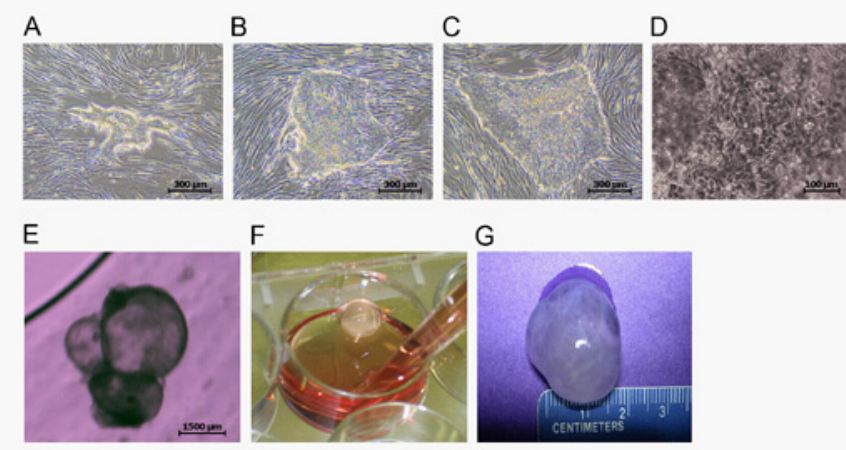

G

Figure 1: Corneal orb differentiation from pluripotent human stem cells.

A. Early appearance of pluripotent stem cell colonies that will go on to form corneal orbs. The small, tightly packed cells in the colony are distinguishable morphologically from the spindle-shaped feeder cells. Scale bar $=300 \mu \mathrm{m}$

B. Proliferation of the pre-cornea colonies results in larger colonies of irregular shapes but with clear edges demarcating the colony from spindle-shaped feeder layer cells. Scale bar $=300 \mu \mathrm{m}$

C. Another expanding colony of differentiating cells easily distinguishable by light microscopy from the feeder layer. Scale bar $=300 \mu \mathrm{m}$

D. Higher power appearance of differentiated pluripotent cells just before the cells are passaged onto low-adherence, deep plates. Scale bar $=100 \mu \mathrm{m}$

E. Differentiating hpSC from which small $(\sim 1-2 \mathrm{~mm})$ orbs have emerged. Scale bar $=1500 \mu \mathrm{m}$

F. Mature corneal orb after 120 days of differentiation is translucent and fluidfilled.

G. Large, mature corneal orb after 120 days of differentiation with diameter of $\sim 15 \mathrm{~mm}$.

of about 4-7 $\mathrm{mm}$. Orbs were generally very fragile until they reached $8-10 \mathrm{~mm}$ in diameter, when they could be manipulated without obvious damage. All orbs were translucent and fluid-filled.

Histological analysis demonstrated that the spheres have interior and outer layers similar in arrangement to normal human cornea. These first histological studies indicated that the epithelium of corneal constructs began as a singlelayer (Figure 2A) and progressively developed additional layers (Figure 1B), following normal developmental patterns. This epithelial layer was PAS-positive indicating glycogen or other polysaccharide content (Figure 2C). PAS stains are used in pathologic evaluation of corneas to visualize corneal epithelial basement membrane (and Descemet's membrane). Histologically the largest layer of the corneal orbs was the stromal layer, morphologically consistent with fibrous tissue. Masson's trichrome staining (Figure 2D) shows that the stromal layer contains collagen fibrils (blue) that were heterogeneous in diameter, some disorganized fibrin strands (pink), and the subepithelial fibrotic membrane, which stained red. (Retention of the red in trichome staining may indicate a poorly permeable structure.) Cytokeratin staining (Figure 2E) was used to further analyze the corneal orbs (using a pan-cytokeratin AE1/3 antibody). Flattened (pancytokeratin-negative) cells were found dispersed between the collagen fibrils, consistent with corneal fibroblasts or keratocytes. The most intense staining was in the superficial epithelial layer (seen at higher power in Figure 2E,F). In addition, in some orbs we identified a thin membrane layer (PAS-negative), between the outer layer of epithelium and the large stromal layer, suggestive of a developing Bowman's membrane but this membrane was not fully formed and was not present in all orbs. The innermost surface of the orbs, where endothelium should be, was a single-cell layer with no evidence of 
keratinization (by pan-cytokeratin staining). Routine hematoxylin and eosin (H\&E) staining was consistent with an endothelium but this stain is non-specific.

Overall, histologic evaluation of these novel stem cell-derived orbs indicated significant structural similarity to human cornea, though a distinct endothelium could not be identified. In addition cultivation of the orbs at an air-liquid interface resulted in thickening of the epithelial layer (comparing Figures 2E and 2F), similar to increased stratification of human corneal epithelium during development at the time of eyelid opening $[17,18]$.

Immunocytochemistry of cornea-like constructs confirmed expression of typical cornea proteins, and normal adult corneas were used to validate the staining pattern. Stem cell-derived corneas expressed mucin-1, a marker of corneal stratified epithelium (and mucin is a component of tears), throughout the epithelial layer (Figure $3 \mathrm{~A}$, normal corneal 3B), and the gap junction protein, connexin-43 (Figure 3C, normal cornea 3D), most strongly by the suprabasal (central) epithelial cells. Cytokeratins (using a pancytokeratin antibody, Figure 3E and normal cornea 3 F) were also expressed, as expected. Specific cytokeratin expression was also examined, and the orbs expressed cytokeratin 19 at low levels (Figure $1 \mathrm{G}$ and normal cornea $1 \mathrm{H}$ ) and cytokeratin 18 (Figure 1I) for which staining was intense only in the superficial layers of the corneal orbs. Strongly positive staining for the tight junction protein ZO-1 was found in all studied specimens, with appropriate
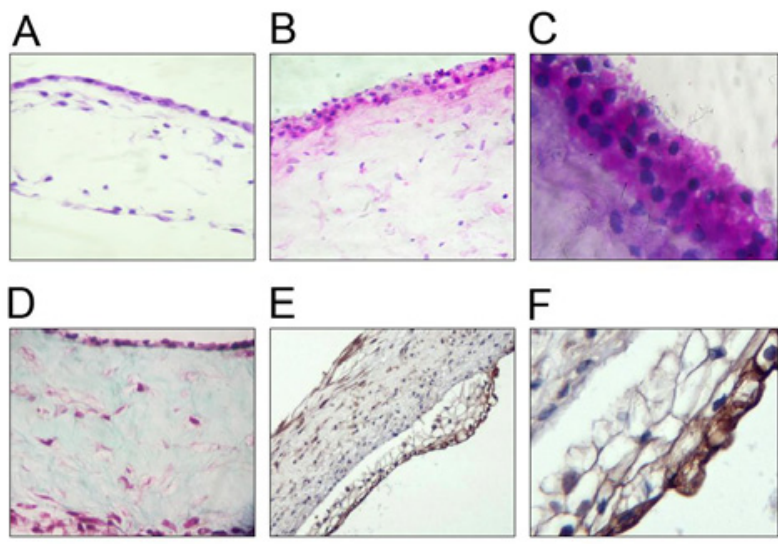

Figure 2: Characterization of multilayered human pluripotent stem cell-derived corneal orbs.

A. H\&E stain of differentiating corneal orb at $\sim 50$ days of differentiation (10X) shows the largest component of corneal construct, the stromal layer, below a single layer of epithelial cells (top of figure).

B. H\&E stain of sectioned corneal orb at the end of 120 days differentiation, showing expansion of the epithelial layer (10X)

C. PAS staining of corneal orb at the end of differentiation shows accumulation of glycogen or other polysaccharides in the epithelium during differentiation (20X)

D. Trichrome staining of corneal orb at end-differentiation shows accumulation of stromal collagen (blue), the major protein of normal corneal stroma. Fibrin strands (pink) are also seen surrounding nuclei of presumed differentiating keratocytes (10X).

E. After exposure to an air-liquid interface, the epithelial layer expands. Brown staining is for cytokeratins (10X).

F. Higher magnification of $(E)$ emphasizes intense cytokeratin staining of the most superficial epithelial cell layer (20X). subcellular localization at cell boundaries suggesting the formation of tight junction complexes, a feature of endothelial cell differentiation (Figure 1J). ZO-1 and connexin-43 staining patterns in the corneal orbs and normal human corneas were very similar, suggesting maturation of intercellular junctions in stem cell-derived orbs. Vimentin staining was positioned in all orbs at low levels, restricted to some stromal cells and the cell layer at the normal anatomic site (innermost) of corneal endothelium (Figure 1K). Stratified epithelial cells were vimentinnegative.

\section{Gene expression of corneal markers}

RT-qPCR assays were used to compare corneal gene expression in fully developed human cornea vs. stem cell-derived corneal orbs. Several genes known to function in all three major layers of the cornea were chosen for study (Figure 4). The expressions of collagen 5 (Col5), decorin, biglycan, lumican, keratocan, CK8, and CK18 in all samples analyzed were significantly higher in stem-cell derived corneas than in adult cornea. The corneal orbs also expressed many characteristic markers of human corneal epithelium including importin 13, integrin alpha 9, connexin-43, and enolase- $\alpha$. Increased expression (vs. adult cornea) of ZO-1, confirming impressions from immunocytochemistry, was also noted in all stem cell-derived corneal orbs.

\section{Drug permeability of corneal orbs}

Permeability and transcorneal transport of drugs are critical processes in pharmacologic treatment of eye disease. To evaluate these functions we performed a small pilot study with two drugs normally delivered topically to the eye. From these studies the permeability coefficient $\left(\mathrm{P}_{\text {app }}\right)$ of the beta-blocker atenolol was calculated to be 2.60 +1.55 (mean + standard deviation) and the $\mathrm{P}_{\text {app }}$ of antipyrine was 15.5 +13.1 . Thus, the low vs. high permeability drugs were transported as expected relative to each other by the stem-cell derived corneas.

\section{Determination of growth factors in differentiating cornea conditioned medium (ELISA)}

Corneal-derived EGF is long known as an important factor in corneal epithelial regeneration [reviewed in 19], while TGF $\beta 1$ has pleiotropic effects in the cornea including maintenance of the stromal layer [20]. Before ninety days of differentiation, neither EGF (in 4/4 samples) nor TGF $\beta 1$ (in $2 / 2$ samples) was detectable using ELISA assays. After that time point, 3 of 4 samples had detectable EGF; the concentrations were $4.3,0.8$, and $2.2 \mathrm{pg} / \mathrm{mL}$ (at the lower limits of detection of the assay). TGF $\beta 1$ was detectable in 3 of 5 samples after 90 days of differentiation; the concentrations were 9.8, 20.6, and $2.8 \mathrm{pg} /$ $\mathrm{mL}$ (at the lower limits of detection of the assay).

\section{Discussion}

The 3D corneal constructs generated in this work emerge as spheres from pluripotent stem cells with several important features of normal cornea including the basic anatomic layering, gene and protein expression patterns, rapid permeability to ophthalmic drugs, and no obvious opacity. This 3D structure represents a significant advance, since generation of functional miniorgans from pluripotent stem cells is very unusual. When induced to differentiate without engineering scaffolds or support, differentiation usually produces random collections of cells in culture, without functional organization. In the case of cornea, the anatomic organization is especially notable, since during normal corneal development, the stroma is derived from neural crest (migrating in to periocular mesenchyme (Hay 1979) whereas local ectoderm (adjacent to lens) becomes corneal epithelium (Cintron, 
Citation: Ostrowska A, Cochran J, Agapova L, Buz'Zard A, Turovets N, et al. (2011) Corneal Spheres derived from Human Embryonic and Human Pluripotent Parthenogenetic Stem Cells. J Stem Cell Res Ther S2:006. doi:10.4172/2157-7633.S2-006
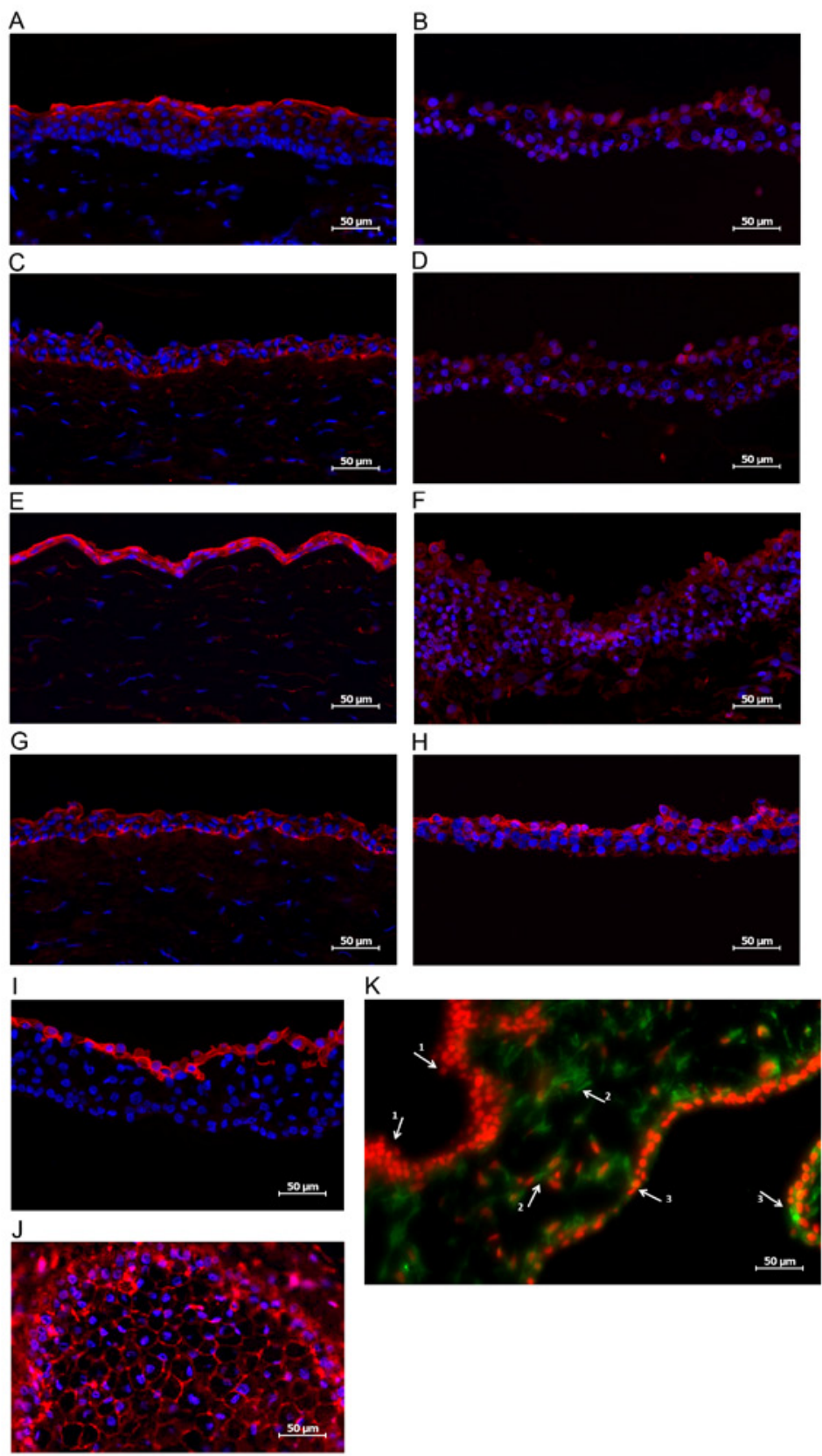

Figure 3: Fluorescent micrographs of immunostaining performed on corneal orbs at the end of differentiation process. DAPI in A-J marks nuclei in blue, propidium iodide in red $(\mathrm{K})$. All scale bars are $300 \mu \mathrm{m}$

A. Mucin-1 immunolstaining (red) of corneal orb concentrated in the epithelial layer is stronger

B. Mucin-1 immunostaining (red) of normal adult human donor cornea.

C. Connexin-43 immunostaining (red) of corneal orb is concentrated on the innermost layer of epithelial cells and overall staining is more intense than

D. Connexin-43 staining (red) of normal adult human cornea.

E. Pancytokeratin immunostaining (red) is intense in the epithelial layer of differentiating corneal orbs (confirming patterns in Figure 2).

F. Less intense and more diffuse pancytokeratin staining (red) in normal human adult cornea than in differentiating orbs.

G. Cytokeratin 19 immunostaining (red) of corneal orbs is intense in the most superficial and deepest epithelial cell layers of the differentiating orbs.

H. Cytokeratin 19 immunostaining (red) is most intense in the most superficial epithelial cell lay of normal human adult cornea.

I. Cytokeratin 18 (red) is abundant in the most superficial epithelial cell layer of differentiating corneal orbs.

J. ZO-1 staining (red) is intense in differentiating corneal orbs with expected subcellular localization to the cell-cell edge interfaces indicating the presence of endothelium.

K. Vimentin negative staining (red) of the multiulayered epithelial cells (arrow 1) and the vimentin positive-staining (green) of differentiating corneal orbs is intense in the entire epithelial stromal layer (interspersed stromal keratocytes, arrow 2) and at the predicted anatomic location of the endothelial monolayer (arrow 3 ). 
Citation: Ostrowska A, Cochran J, Agapova L, Buz'Zard A, Turovets N, et al. (2011) Corneal Spheres derived from Human Embryonic and Human Pluripotent Parthenogenetic Stem Cells. J Stem Cell Res Ther S2:006. doi:10.4172/2157-7633.S2-006

\section{A}
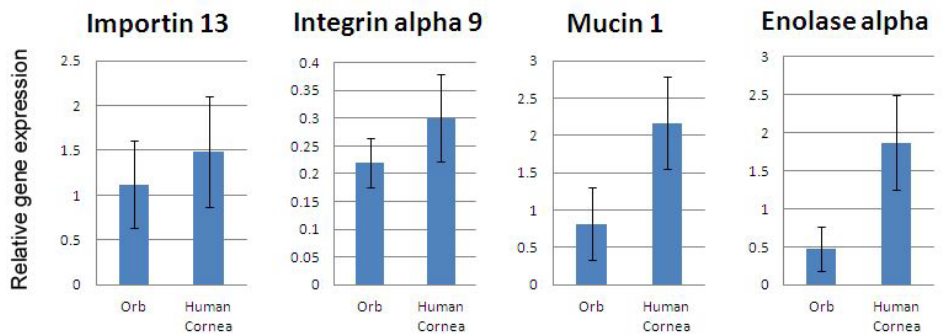

\section{B}

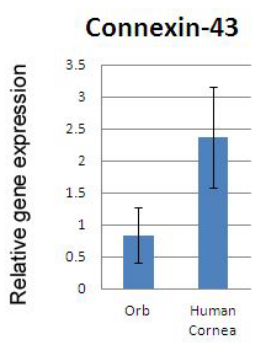

ZO-1

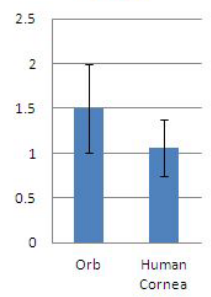

C

CK8

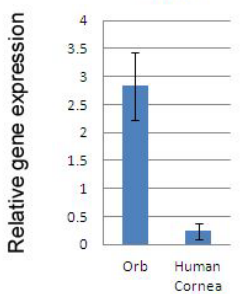

D

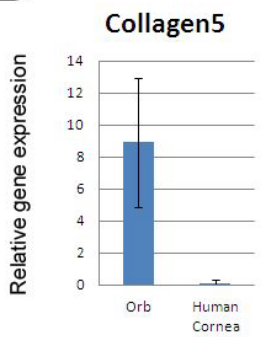

CK10

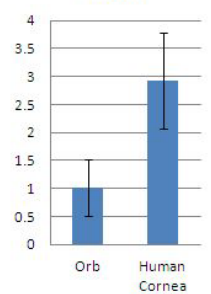

Decorin

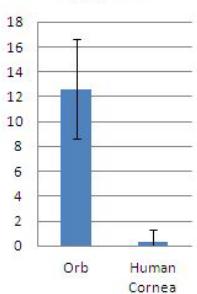

Biglycan

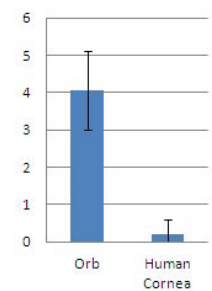

Lumican

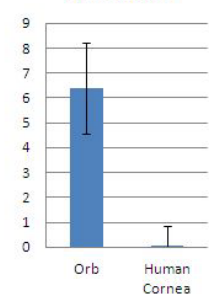

Keratocan

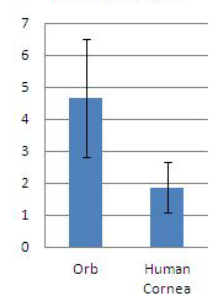

Figure 4: Trends in relative gene expression of corneal genes in differentiating corneal orbs ( $n=3$ independent orbs) vs. adult human cornea ( $n=2$ independent corneas) show that the differentiating orbs express many normal corneal genes. Data are expressed as mean and bars are standard deviation. (Statistics not performed because of low $\mathrm{n}$ and high S.D.)

A. Importin 13 may be expressed by a resident limbal stem cell population in both differentiating and normal adult corneas, but the limbal stem cell population was not assayed with other markers. Integrin alpha 9, connexin-43, and enolase- $\alpha$ are normally expressed in corneal epithelium,

B. Connexin-43 and ZO-1 are both important for cell-cell communication in the cornea, and combined with immunohistochemistry (Figure 3 ) these results suggest maturation of subcellular structures involved in intercellular communication by 120 days of differentiation.

C. Differential expression of cytokeratins suggests relatively high expression of CK8 and CK19 in the stem cell-derived differentiating corneal orbs relative to mature adult cornea.

D. The most striking pattern in the gene expression studies was the high expression of various extracellular matrix proteins in the differentiating orbs compared to the lower level expression in mature adult cornea. At the protein level these ECM genes are low abundance in normal cornea compared to the relatively large collagen content of cornea. 
1983), yet both layers were generated in a single differentiation protocol from pluripotent stem cells. Using the conditions we describe, generation of the epithelial layer in the corneal orbs appears to be robust by histology, by functional manipulation of the epithelium at an air-liquid interface, supported by expression of multiple developing epithelial genes (cytokeratins 8,10 , and 18 , mucin-1, connexin-43, and enolase- $\alpha$ ). Generation of the large stromal layer is also consistent, as seen histologically, by PAS staining, and gene expression (decorin, lumican, biglycan, and keratocan). The stroma appears to be quite differentiated and lacked expression of the early stromal marker, Pax6. Some optimization is likely required for the endothelial layer, which may require longer differentiation (or an added differentiation step) to develop. In some corneal orbs we identified cells histologically that were appropriately localized and shaped for endothelial cells. Vimentin and zona occludens staining (expressed during endothelial development) also indicate some endothelial cells in the orb interior, but we cannot yet determine if endothelium is reliably incorporated into the corneas at 100-120 days of differentiation. We have not yet quantified the number of limbal stem cells in the differentiating corneas, but their presence is suggested by the expression of importin-13 (IPO13) characteristic of corneal epithelial progenitor cells. In normal cornea, IPO13 is uniquely expressed by human limbal basal epithelial cells, and plays an important role in maintaining the progenitor phenotype and high proliferative potential [21].

In a small pilot study we examined permeability of the corneal orbs. The primary pathway of drug permeation from the surface tear fluid to the anterior chamber of the eye is transcorneal [22] and passage through the corneal epithelium is considered to be the rate-limiting step in the transcorneal penetration of most ophthalmic drugs. Our results suggested rapid corneal permeability of the two drugs tested and as well as the expected greater permeability of antipyrine over atenolol. Some secretory function of the corneas was confirmed at the later stages of differentiation. EGF, which stimulates corneal epithelial cell proliferation and migration [reviewed in 23] and TGF $\beta 1$, involved in maintaining corneal integrity, in part by counterbalancing the stimulatory effects of EGF [reviewed in 24] were detectable in medium around the developing corneas. Both EGF and TGF $\beta 1$ promote keratocyte differentiation [25] and chemotaxis [26].

Though the generation of a 3D cornea from a single cell source rather than via multiple differentiation procedures is somewhat surprising and promising for clinical manufacture, the pluripotent stem cell-derived corneas will also require further manipulation to optimize them for preclinical studies, particularly to perfect the alignment of collagen in the stromal layer to assure optimal refractive properties [27]. Normal corneal fibroblasts produce collagen fibrils of uniform size and spacing, which are then orthogonally arranged in the stroma matrix [28], and changes to this pattern of organization result in loss of corneal transparency. Finally, though the corneal spheres appear to be transparent, their true transparency needs to be quantified by physical measures.

The differentiation process yields corneal orbs with sufficient integrity that we anticipate they can be manipulated in engineering studies and moved to other culture (or co-culture) environments for further differentiation. The orbs are also suitable, after some further development, for combination with various bioengineered corneal materials in a 'combined device-cell therapy' approach taken by other groups $[6,29]$. These are all research agendas we are currently investigating, to take advantage of recent progress in generating biosynthetic corneas. Among the most promising of these biosynthetic corneas, a recent Phase I trial of cross-linked collagen corneal grafts were implanted into ten patients (9 with keratoconus) who had intact corneal endothelium. The grafts induced endogenous reepithelialization over the course of about a month, re-innervation starting at about a year, supported normal tear formation, without prolonged immunosuppression and without pain. Most of the treated patients could tolerate contact lenses to improve vision, which was not as good as the vision of comparable patients who received full thickness human allogeneic grafts [8].

The smaller size (though similar organization) of corneal orbs generated from hpSC compared to those generated from hESC will require the protocol for $\mathrm{hpSC}$ undergo modification relative to the protocol used with hESC. We have reported other differences in growth and differentiation patterns between hpSC and hESC lines. For example, when a protocol that yields efficient ( $\sim 80 \%)$ differentiation of hESC into definitive endoderm [30] is applied to hpSC, the efficiency is about half that of hESC. But pre-treatment of the hpSC with trichostatin A increases the efficiency ( $75 \%)$ to that of hESC differentiation [31]. The yield of neural progenitor cells from hpSC is also less than from hESC, which may depend in part on differences in expression of extracellular matrix proteins necessary to hold neurospheres together during differentiation [32]. Adaptation of protocols that do not require neurospheres is therefore a rational choice for hpSC. Differentiation of retinal pigment epithelial (RPE) cells from hpSC and hESC, accomplished without an intermediate sphere formation step, yields similar numbers of qualitatively similar RPE [32].

The focus on parthenogenetic stem cells as a source for corneas (and other tissues), is well-justified by the clinical literature on the immunogenicity of allogeneic corneal grafts. Rejection of cornea allografts is a major cause of graft failure [33], and a history of rejection puts patients at high risk of future (second) graft loss [34]. HLA matching can improve corneal allograft survival [35] but is not routinely done, based in part on cost, and because there is not global consensus on its value. Nonetheless, the major cause of graft failure in the first year after corneal transplantation is cell-mediated rejection [36]. A bank of HLA homozygous parthenogenetic stem cells to cover common HLA haplotypes in the population is, therefore, a potentially valuable source of transplantable cells that can be better matched to reduce the intensity and incidence of rejection.

In summary, we generated free-floating corneal orbs from both human ESC and smaller orbs from parthenogenetic pluripotent stem cells, and these corneas differentiate as a 3D layered structure similar to that of normal human corneas. A priority for continued pre-clinical development of these corneas is engineering approaches to improve stromal collagen organization and on developing variances in culture protocols aimed at increasing the size of hpSC-genreated orbs. The clinical need for corneal grafts for blinding diseases, especially outside the U.S., is great, highlighting the importance of further research to optimize these potential stem cell-derived grafts.

\section{Acknowledgments}

The authors want to thank Dr. Mike West for providing hESC line WA09 for this research and Dr. Chris Bode (Absorption Systems, Exton, PA) for his technical support and advice.

\section{References}

1. Whitcher JP, Srinivasan M, Upadhyay MP (2001) Corneal blindness: a global perspective. Bull World Health Org 79: 214-221. 
Citation: Ostrowska A, Cochran J, Agapova L, Buz'Zard A, Turovets N, et al. (2011) Corneal Spheres derived from Human Embryonic and Human Pluripotent Parthenogenetic Stem Cells. J Stem Cell Res Ther S2:006. doi:10.4172/2157-7633.S2-006

2. Lagali N, Fagerholm P, Griffith M (2011) Biosynthetic corneas: prospects for supplementing the human donor cornea supply. Expert Rev Med Devices 8: 127-130.

3. Krachmer JH, Mannis MJ, Holland EJ (2011) Cornea, $3^{\text {rd }}$ edition, Elsevier, New York NY.

4. Ahmad S, Figueiredo F, Lako M (2006) Corneal epithelial stem cells: characterization, culture and transplantation. Regen Med 1: 29-44.

5. Rama P, Matuska S, Paganoni G, Spinelli A, De Luca M, et al.(2010) Limba stem-cell therapy and long-term corneal regeneration. N Engl J Med 363: 147155.

6. Builles N, Justin V, Andre V, Burillon C, Damour O (2007) Reconstructed corneas: effect of three-dimensional culture, epithelium, and tetracycline hydrochloride on newly synthesized extracellular matrix. Cornea 26: 12391248.

7. McLaughlin CR, Tsai RJ, Latorre MA, Griffith M (2009) Bioengineered corneas for transplantation and in vitro toxicology. Front Biosci 14: 3326-3337.

8. Fagerholm P, Lagali NS, Merrett K, Jackson WB, Munger R, et al. (2010) A biosynthetic alternative to human donor tissue for inducing corneal regeneration: 24-month follow-up of a Phase 1 clinical study. Sci Transl Med 2: 46ra61.

9. McIntosh AW, Schein O, Elisseeff J (2010) A tale of two tissues: stem cells in cartilage and corneal tissue engineering. Curr Stem Cell Res Ther 5: 37-48.

10. Barbaro V, Ferrari S, Fasolo A, Ponzin D, Di lorio E (2009) Reconstruction of a human hemicornea through natural scaffolds compatible with the growth of corneal epithelial stem cells and stromal keratocytes. Mol Vis 15: 2085-2093.

11. Proulx S, d'Arc Uwamaliya J, Carrier P, Deschambeault A, Audet C, et al (2010) Reconstruction of a human cornea by the self-assembly approach of tissue engineering using the three native cell types. Mol Vis 16: 2192-2201.

12. Ahmad S, Stewart R, Yung S, Kolli S, Armstrong L, et al. (2007) Differentiation of human embryonic stem cells into corneal epithelial-like cells by in vitro replication of the corneal epithelial stem cell niche. Stem Cells 25: 1145-1155.

13. Revazova ES, Turovets NA, Kochetkova OD, Kindarova LB, Kuzmichev LN, et al. (2007) Patient-specific stem cell lines derived from human parthenogenetic blastocysts. Cloning Stem Cells 9: 432-449.

14. Revazova ES, Turovets NA, Kochelkova OD, Agapova LS, Sebastian JL, et al. (2008) HLA homozygous stem cell lines derived from human parthenogenetic blastocysts. Cloning Stem Cells 10: 11-24.

15. Turovets N, Semechkin A, Kuzmichev L, Janus J, Agapova L, et al.(2011) Derivation of human parthenogenetic stem cell lines. Methods Mol Biol 767: 37-54.

16. Gaudana R, Ananthula HK, Parenky A, Mitra AK (2010) Ocular drug delivery. AAPS J 12: 348-360.

17. Chung EH, Bukusoglu G, Zieske JD (1992) Localisation of corneal epithelial stem cells in the developing rat. Invest Ophthalmol Vis Sci 33: 2199-2206.

18. Watanabe H, Tisdale AS, Gipson IK (1993) Eyelid opening induces expression of a glycocalyx glycoprotein of rat ocular surface epithelium. Invest Ophthalmo Vis Sci 34: 327-338.

19. Schultz G, Chegini N, Grant M, Khaw P, MacKay S (1992) Effects of growth factors on corneal wound healing. Acta Ophthalmol Suppl 202: 60-66.

20. Carrington LM, Albon J, Anderson I, Kamma C, Boulton M (2006) Differential regulation of key stages in early corneal wound healing by TGF-beta isoforms and their inhibitors. Invest Ophthalmol Vis Sci 47: 1886-1894.

21. Wang H, Tao T, Tang J, Mao YH, Li W, et al. (2009) Importin 13 serves as a potential marker for corneal epithelial progenitor cells. Stem Cells 27: 25162526.

22. Xiang CD, Batugo M, Gale DC, Zhang T, Ye J, et al. (2009) Characterization of human corneal epithelial cell model as a surrogate for corneal permeability assessment: metabolism and transport. Drug Metab Dispos 37: 992-998.
23. Zelenka PS, Arpitha P (2008) Coordinating cell proliferation and migration in the lens and cornea. Semin Cell Dev Biol 19: 113-124.

24. Tandon A, Tovey JC, Sharma A, Gupta R, Mohan RR (2010) Role of transforming growth factor Beta in corneal function, biology and pathology. Curr Mol Med 10: 565-578.

25. He J, Bazan HE (2008) Epidermal growth factor synergism with TGF-beta1 via $\mathrm{PI}-3$ kinase activity in corneal keratocyte differentiation. Invest Ophthalmol Vis Sci 49: 2936-2945.

26. Andresen JL, Ehlers N (1998) Chemotaxis of human keratocytes is increased by platelet-derived growth factor-BB, epidermal growth factor, transforming growth factor-alpha, acidic fibroblast growth factor, insulin-like growth factor-I and transforming growth factor-beta. Curr Eye Res 17: 79-87.

27. Ruberti JW, Zieske JD (2008) Prelude to corneal tissue engineering - gaining control over collagen organization. Prog Retin Eye Res 27: 549-577.

28. Jester JV, Winkler M, Jester BE, Nien C, Chai D, et al. (2010) Evaluating corneal collagen organization using high resolution non linear optical (NLO) macroscopy. Eye Contact Lens 36: 260-264.

29. Shah A, Brugnano J, Sun S, Vase A, Orwin E (2008) The development of a tissue-engineered cornea: biomaterials and culture methods. Pediatr Res 63 535-544.

30. D'Amour KA, Agulnick AD, Eliazer S, Kelly OG, Kroon E, et al. (2005) Efficient differentiation of human embryonic stem cells to definitive endoderm. Nat Biotechnol 12: 1534-1541.

31. Turovets N, D’Amour KA, Agapov V, Turovets I, Kochetkova O, et al.(2011) Human parthenogenetic stem cells produce enriched populations of definitive endoderm cells after trichostatin A pretreatment. Differentiation 81: 292-298.

32. Harness JV, Turovets N, Seiler MJ, Nistor G, Altun G, et al. (2011) Equivalence of conventionally-derived and parthenote-derived embryonic stem cells. PLoS One 6(1): e14499.

33. Coster DJ, Williams KA (2005) The impact of corneal allograft rejection on the long-term outcome of corneal transplantation. Am J Ophthalmol 140: 1112 1122.

34. Joseph A, Raj D, Shanmuganathan V, Powell RJ, Dua HS (2007) Tacrolimus immunosuppression in high-risk corneal grafts. $\mathrm{Br} \mathrm{J}$ Ophthalmol 91: 51-55.

35. Bohringer D, Spierings E, Enczmann J, Bohringer S, Sundmacher R, et al (2006) Matching of the minor histocompatibility antigen $\mathrm{HLA}-\mathrm{A} 1 / \mathrm{H}-\mathrm{Y}$ may improve prognosis in corneal transplantation. Transplantation 82: 1037-1041.

36. Armitage WJ (2004) HLA matching and corneal transplantation. Eye (Lond) 18: 231-232.

37. Gonzalez-Andrades M, de la Cruz Cardona J, Ionescu AM, Campos A, Del Ma Perez M, et al. (2011) Generation of bioengineered corneas with decellularized xenografts and human keratocytes. Invest Ophthalmol Vis Sci 52: 215-222.

38. Lampert KP (2008) Facultative parthenogenesis in vertebrates: reproductive error or chance? Sex Dev 2: 290-301.

39. Mitalipov SM, Nusser KD, Wolf DP (2001) Parthenogenetic activation of rhesus monkey oocytes and reconstructed embryos. Biol Reprod 65: 253-259. 